\title{
Saskia Esken* \\ Für eine offene Wissensgesellschaft: Digitales Publizieren und Open Access
}

DOI 10.1515/bfp-2016-0037

Zusammenfassung: Der Beitrag plädiert für ein Bündnis zwischen Hochschulen, wissenschaftlichen Autoren, Bibliotheken, Verlagen und den Nutzenden mit dem Ziel einer Verbesserung des Zugangs zu Wissen. Freier Zugang, d.h. Open Access, zu wissenschaftlichen Publikationen wie auch zu Forschungsdaten sind ein wesentlicher Antrieb für die Weiterentwicklung der modernen Wissensgesellschaft.

Schlüsselwörter: Digitalisierung; Informationskompetenz; Open Access; Horizon 2020; Wissensgesellschaft

\section{Towards an Open Information Society: Digital Publishing and Open Access}

Abstract: The article argues for a new alliance between universities, scientific authors, libraries, publishers, and the users aiming at improving the access to knowledge. Open Access to scientific publications but also to research data forms an essential drive towards the further advancement of the information society.

Keywords: Digital transformation; information literacy; open access; Horizon 2020; information society

Mit der Digitalisierung und den damit verbundenen Entwicklungen und Errungenschaften eröffnen sich neue Möglichkeiten. Heute können wir - über nationale, kulturelle, aber auch über hierarchische Grenzen hinweg mit aller Welt kommunizieren. Wir vernetzen uns und arbeiten in virtuellen Teams, wie wir uns das noch vor 20 Jahren nicht vorstellen konnten. Der ungehinderte Zugang zu Wissen und die Kompetenz im Umgang damit - manche nennen es Informationskompetenz - zählen zu den wichtigsten Voraussetzungen für gesellschaftliche Teilhabe.

Auf den ersten Blick scheint es so, als verfügten wir heute über eine - im Vergleich zu früher - ungeheure Menge und Vielfalt an Informationen. Sobald es aber um gesicherte Informationen und wissenschaftlich geprüfte Daten geht, ist ein offener digitaler Zugang jedoch vielfach

*Kontaktperson: Saskia Esken, MdB, saskia.esken@bundestag.de noch nicht vorhanden. Der Weg zu dem aktuellsten Wissen aus Forschung und Wissenschaft ist mit hohen Hürden verbunden.

Noch immer werden wissenschaftliche Erkenntnisse durch die Veröffentlichung bei renommierten Verlagen nur einem gut zahlenden Publikum zugänglich gemacht. Im Rahmen dieser geschlossenen Lizenzen ist eine Zweitveröffentlichung nicht erlaubt. Obwohl wissenschaftliche Erkenntnisse durch die Förderung aus öffentlichen Mitteln erst ermöglicht werden, sind sie für interessierte Bürger von außerhalb der Hochschule nicht zugänglich. Selbst die Hochschulen und ihre Bibliotheken können und wollen die enormen Summen für wissenschaftliche Zeitschriften oft nicht mehr ausgeben. Ohne Austausch und Vernetzung bleibt Wissenschaft aber viel zu oft unfruchtbar.

Auch die für wissenschaftliche Arbeiten erhobenen Daten werden gehütet wie ein Schatz. Anders als bei materiellen Reichtümern erhöht sich der Wert dieser Datenschätze noch um ein Vielfaches, wenn man die einmal erhobenen Daten teilt. Durch den Vergleich mit anderen Perspektiven erhöht sich das Vertrauen in die Analyse und es werden neue Zusammenhänge deutlich. Mit jeder zusätzlichen Verwendung und Auswertung in ähnlichen oder ganz unterschiedlichen wissenschaftlichen Kontexten ergibt sich ein Gewinn an Wissen.

Angesichts dieser Vorteile ist es nicht überraschend, dass der kostenfreie und offene Zugang zu wissenschaftlichen Erkenntnissen und Daten als Open Access und Open Data immer mehr in den Vordergrund rückt. Andere Länder sind bei der Öffnung von Daten allerdings schon viel weiter: 2013 wurde in den USA unter Präsident Obama die Verpflichtung zu Open Access im Rahmen der Forschungsförderung verankert. Auch die Europäische Union hat sich zum Ziel gesetzt, dass bis 2016 mindestens 60 Prozent aller Ergebnisse aus öffentlich geförderter Forschung frei zugänglich sein sollten. Im aktuellen Rahmenprogramm der EU zur Förderung von Forschung und Innovation, Horizon 2020, ist die Open-Access-Veröffentlichung für alle wissenschaftlichen Publikationen verpflichtend.

Als Pionier in der EU muss Großbritannien gelten. Bereits 2004 hat das Britische Unterhaus ein Zweitveröffentlichungsrecht für wissenschaftliche Autoren empfohlen, damit diese ihre Artikel in öffentlich zugänglichen Repositorien zur Verfügung stellen können. Mittlerweile 
gibt es in Großbritannien eine große Zahl solcher Repositorien und damit einen hohen Anteil öffentlich zugänglicher wissenschaftlicher Arbeiten.

In Deutschland werden die Ergebnisse wissenschaftlicher Arbeit traditionell in renommierten privatwirtschaftlichen Fachjournalen veröffentlicht und insbesondere in den Geisteswissenschaften hat das dort gedruckte Wort eine geradezu symbolische Bedeutung. Neben der Zitation in anderen Werken gilt bereits der Ort der Publikation als Nachweis wissenschaftlicher Qualität und wirkt sich auf die gewünschte Anerkennung aus. Einer Open-Access-Veröffentlichung in traditionellen, aber ausschließlich online erscheinenden Zeitschriften wird diese Anerkennung in den Geisteswissenschaften leider noch nicht im gleichen Maße entgegengebracht - völlig zu Unrecht, wie Auswertungen zur Zitierhäufigkeit von Open-Access-Artikeln regelmäßig zeigen.

Dennoch hat neben der Onlinepublikation auch die Wahrnehmung und Anerkennung der traditionellen Verlagsveröffentlichung ihre Begründung, welche beim Aufbau einer zukunftsfähigen Wissenschaftskultur zu berücksichtigen ist. Diese Herausforderung lässt sich nicht ausschließlich durch politisches Handeln lösen: Auch die Wissenschaftskultur muss an ihrer Praxis der Anerkennung arbeiten. Gemeinsam sollten wir soweit kommen, dass zumindest die digitale Zweitveröffentlichung als Open Access mehr und mehr zur Regel wird. Denn bei Open Access gibt es eigentlich nur Gewinner.

Während die Autoren wissenschaftlicher Publikationen für die Wahrnehmung und Anerkennung durch die ausschließliche Veröffentlichung bei einem renommierten Verlag mit der vollständigen Abtretung ihrer Rechte bezahlen, finden sie durch die Zweitveröffentlichung neue Leser sowohl unter Kollegen als auch darüber hinaus. Die Publikation wird durch eine frühe elektronische Verfügbarkeit (z.B. als pre-print) schneller und häufiger durch Kollegen zitiert und gewinnt an Sichtbarkeit und Reputation.

Von einem offenen Zugang zu wissenschaftlichen Erkenntnissen und Daten profitiert die gesamte Gesellschaft. Open Access fördert den wissenschaftlichen Austausch und erlaubt einen breiten Diskurs über die Grenzen einzelner Fächer hinaus. Nicht nur Angehörige einer Hochschule, sondern auch die breite Öffentlichkeit kann sich über Suchmaschinen, Weblogs und Diskussionsplattformen, Wikis und soziale Netzwerke über die neuen Erkenntnisse informieren und austauschen.

Die SPD-Vertreter haben deshalb in den Verhandlungen zur großen Koalition dafür gesorgt, dass im Koalitionsvertrag die folgende Vereinbarung mit aufgenommen wurde: „Wir werden eine umfassende Open-Access-Strategie entwickeln, die die Rahmenbedingungen für einen effektiven und dauerhaften Zugang $\mathrm{zu}$ öffentlich finanzierten Publikationen und auch zu Daten (Open Data) verbessert.“

Wir setzen uns deshalb auch dafür ein, dass das Bundesministerium für Bildung und Forschung seine Mittelvergabe künftig von einer Open-Access-Veröffentlichung abhängig macht. Die Zielsetzung der Europäischen Union müssen wir uns zu Eigen machen und unsere Devise muss dabei lauten: Wer öffentliche Gelder für die Forschung erhält, der soll auch öffentlich publizieren.

Gerade die außeruniversitären Forschungseinrichtungen sind im Open-Access-Bereich oft schon sehr weit. Die Fraunhofer-Gesellschaft, die Helmholtz-Gemeinschaft, die Leibniz-Gemeinschaft und die Max-Planck-Gesellschaft verfolgen eigene Open-Access-Ziele. Diese Entwicklung halte ich für sehr positiv. Mittelfristig will die SPD-Bundestagsfraktion die Finanzierung auch der außeruniversitären Forschungseinrichtungen davon abhängig machen, dass die veröffentlichten Ergebnisse nach einer Frist von maximal zwölf Monaten frei zugänglich gemacht werden.

Auch eine Veröffentlichung mit freiem Zugang ist nicht kostenlos machbar. Wissenschaftliche Publikationen verursachen durch die Notwendigkeit zur Qualitätssicherung erhebliche Kosten und das gilt ebenfalls für die Veröffentlichung in Open Access. Die SPD-Bundestagsfraktion schlägt deshalb vor, für die Finanzierung von Open Access einen Fonds einzurichten, aus dem die Publikationsgebühren bei renommierten Medien insbesondere für Nachwuchswissenschaftler gefördert werden können. Die Vereinbarung einheitlicher Schnittstellen und die Entwicklung einer gemeinsamen Software sollen zudem die technischen Entwicklungskosten im Open-Access-Bereich gering halten.

Ich bin der festen Überzeugung, dass es möglich ist, ein neues Bündnis zwischen Hochschulen, wissenschaftlichen Autoren, Bibliotheken, Verlagen und der Leserschaft zu schließen. Lassen Sie uns ein solches Bündnis gemeinsam anstreben, damit bei uns in Deutschland und darüber hinaus eine demokratische und vernetzte Wissensgesellschaft entstehen kann.

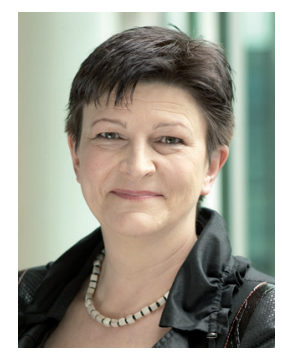

Saskia Esken, MdB Deutscher Bundestag Platz der Republik 1 D-11011 Berlin saskia.esken@bundestag.de 